\title{
Coordinated Intelligent Traffic Control System (CITCS)
}

\author{
Bhupinder Singh Saini \\ Lovely Professional University, \\ Phagwara, India
}

\author{
Ankur Sodhi \\ Lovely Professional University, \\ Phagwara, India
}

\author{
Baljit Singh Saini \\ Lovely Professional University \\ Phagwara, India
}

\begin{abstract}
This research aims in providing an efficient way to detect, control and manage the traffic and the traffic problems at the lanes without the involvement of any traffic police or the traffic management staff. This research finishes the human involvement completely. This system also provides the congestion control mechanism. This research overcomes drawbacks of other techniques developed earlier like Inductive Loop Detection, Video Data Analysis etc. The present intelligent system will consist of the RFID readers placed at the start of the lane and the end of the lane that is near the traffic lights. The readers placed at the end of the lane will measure the traffic at the end of the lane and the readers at the start of the lane will measure the upcoming traffic and they will also detect the emergency vehicles, (if any), plus they will also contribute in the congestion control mechanism. The traffic lights will work on priority basis dynamically. This research believes in saving the time of the vehicles at the cross roads where the traffic lights have been installed.
\end{abstract}

\section{Keywords}

Intelligent system, control unit, RFID sensors, congestion, static, dynamic

\section{INTRODUCTION}

This research proposes a well design for a Coordinated Intelligent Traffic Control System. This research shows the importance in the cities which have high traffic of vehicles on roads. This proposed system is designed in such a way that it will greatly reduce the traffic problems and will save the time of the people at the Traffic Lights as well as who are approaching the Traffic Lights and also it will control the congestion at the roads. There have been many previous researches done like SCOOT system that has been implemented in UK [1]. There are other systems also which have been designed with the purpose of providing the valuable information to the driver for choosing the path [2]. Some controls the switching operation of the system [3], [4]. Some tracks the amount of traffic by capturing the images [5], [6]. The recent studies focused on applications of RFID [7].

\section{RESEARCH PROBLEMS}

After completely studying the working of traffic lights in most of the heavily crowded cities, the following problems have been noticed:

- The roads get jammed with high number of vehicles in the rush hours which leads to the wastage of the time as well as the fuel at the traffic lights.

- The current traffic control systems don't have the power of giving longer tome period to the high congestion lanes depending upon the traffic present at the lane.

\begin{abstract}
- When the traffic level leads to bottleneck, the traffic system is turned to manual mode and the traffic officer is called to control the traffic manually.
\end{abstract}

- When there is a green signal at the lane instead there is no traffic leads to the wastage of time of the people at the other lanes.

\section{OBJECTIVES}

- Controlling the traffic jams on roads due to increase in the number of vehicles.

- Saving the time of the people at the traffic lights.

- Providing the priority to the VIP and the emergency vehicles like ambulances which want a clear way ahead to pass through.

- Controlling the congestion problems occurred due to the scenarios like accidents.

- Saving the time of the vehicles approaching the traffic lights if they can pass through.

- What to do when one ambulance and one VIP vehicle arrives at the same time in two different lanes.

- What to do when two ambulances arrive in two different lanes simultaneously.

\section{PROPOSED CITCS MODEL}

The proposed model consists of the following main components to work with.

\subsection{Main components of the proposed model}

\subsubsection{Central control unit}

This unit is installed at the Main Traffic Control Station. Every pair of traffic lights has its separate control unit. This unit receives all the information from the RFID readers and possesses the power to process it and make the decisions accordingly. It makes the following four decisions:

- For how long the green signal is to be provided to a particular lane?

- Which lanes will have the high priority?

- Is there any congestion at the traffic lanes or not?

- Is there any emergency vehicle in the lane?

\subsubsection{RFID readers}

These readers sense the RFID Tags information that are embedded in the vehicles. 


\subsubsection{RFID tags}

These tags are embedded in the vehicles. Each tag has a unique identification number. The special vehicles that is the VIP and the Emergency vehicles have special tag numbers which help in their detection.

\subsubsection{Traffic lights}

These are connected to the Control Unit and work according to the control signals received from the Control Unit.

\section{The probability distribution of cars and the assumptions}

\subsubsection{Probability distribution of cars}

The first level $S_{0}$ to $S_{1}$ shows the traffic in the normal state, $S_{1}$ to $S_{2}$ represents the traffic when there is moderate traffic, and $S_{2}$ to $S_{3}$ represents the traffic when there is traffic congestion as in fig. 1 [8].

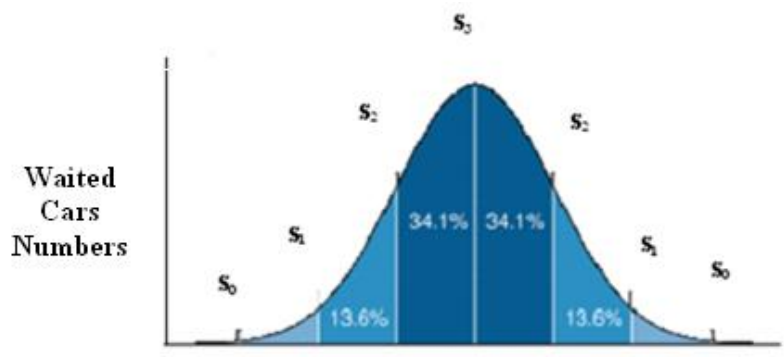

Time

Figure 1: Time vs. no. of waited cars

\subsubsection{Assumptions}

- The traffic goes in a disciplinary way.

- One vehicle takes ' $\alpha$ ' seconds to cross the Traffic Light Junction.

\section{WORKING OF THE PROPOSED MODEL}

The RFID readers are placed at the end as well as at the start of the lane as shown in figure 2.

- $R_{1} E, R_{2} E, R_{3} E$ and $R_{4} E$ are the RFID readers at the end of the lanes $L_{1}, L_{2}, L_{3}$ and $L_{4}$. In the same way, $R_{1} S, R_{2} S, R_{3} S$ and $R_{4} S$ are the RFID readers at the RFID readers at the start of the lanes $L_{1}, L_{2}, L_{3}$ and $L_{4}$ respectively.

- The readers at the end of the lanes will pass the information about the number of vehicles (say $n_{1}$,) that are in their range to the Control Unit.

- Similarly, the readers at the start of the lane will pass the information about the number of vehicles passing (say $n_{2}$ ) through their range to the Control Unit till the traffic light for that lane is not green.



Figure 2: The proposed model

\subsection{Normal case}

The time of the green light will be

$\operatorname{if}\left(T^{\prime}(m) \leq T(m) \& \& T^{\prime}(m) !=G R E E N\right)$

\{

Case 2;

\}

else

\{

Case 1;

\}

Where,

- $\mathrm{m}$ is the lane number $-1,2,3$ or 4 .

- $T^{\prime}(m)$ is the time taken by vehicles to reach at the traffic lights, given by

$T^{\prime}(m)=D(m) / S$, where $\mathrm{D}$ is the length of the lane that is the distance that a vehicle has to travel to reach the traffic lights of that lane and $\mathrm{S}$ is the speed allowed at that particular lane, assumed to be the speed of the vehicle.

- $T(m)$ is the time left for the green light is be turned on.

Case 1: $T(m)=n_{1} * \alpha+\beta$, where ' $\beta$ ' is the extra leverage time.

Case 2: $T(m)=n_{1} * \alpha+n_{2} * \alpha+\beta$ 


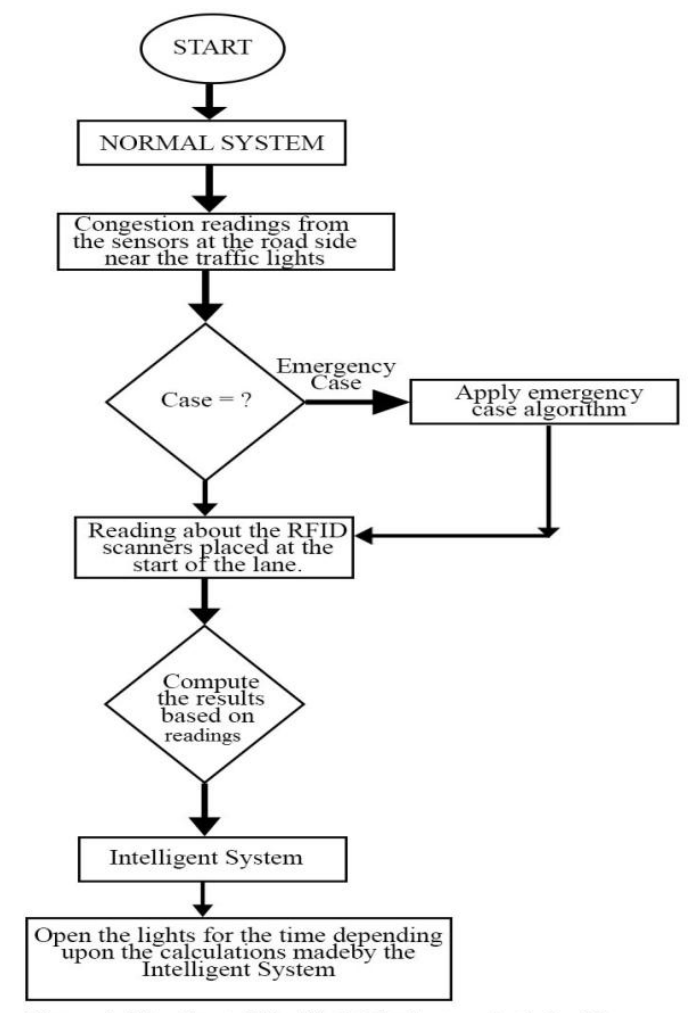

\subsection{Emergency case}

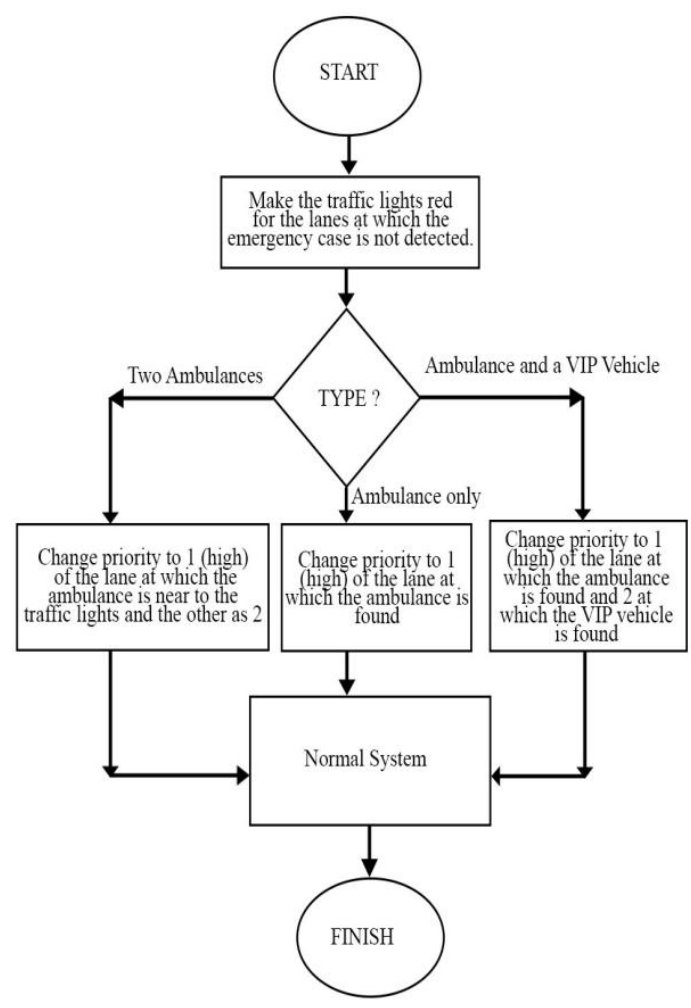

Figure 4: The Emergency Case Activity Diagram

\subsection{Congestion control mechanism}

The congestion mechanism works in the following fashion as the steps of the algorithm:

1) Assume the time taken by the vehicle to be $\mathrm{x}$ seconds to cover the distance from the start to end of the lane.

2) A passkey is assigned to the vehicles which changes after every $\mathrm{x}$ seconds at both the start and the end terminal RFID readers.

3) Assign the permanent passkey be y to the vehicle.

4) Map that passkey with the RFID reader's packets at the start and at the end terminals.

5) If $y$ of the vehicle is same as the $y$ of the end terminal RFID reader, then : no congestion

6) Else : there is a congestion or a jam.

7) Information for the adjusting the time of lights to central

\section{RESULTS}

In order to get the results, the following two assumptions have been made:

- ' $\alpha$ ' is supposed to be 1 second.

- 'static' represents the cases without RFID implementation and 'dynamic' represents the cases with RFID implementation.

\subsection{Time saved}

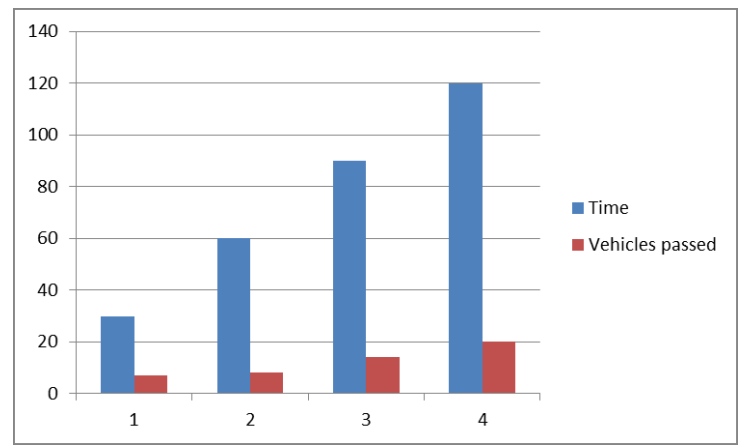

Figure 5. Time vs. number of vehicles without RFID

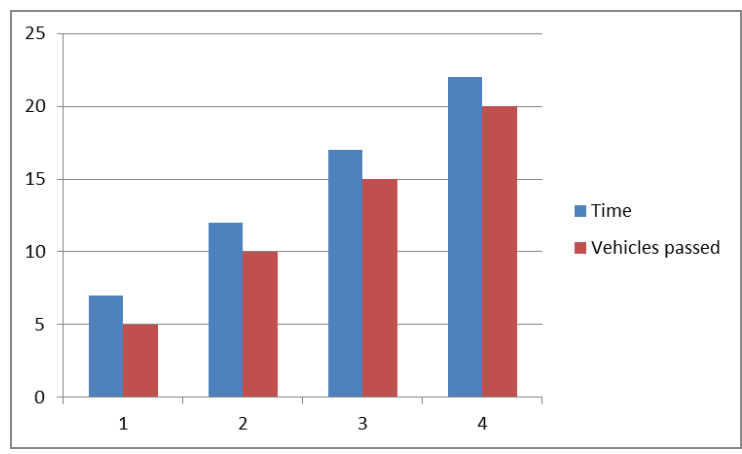

Figure 6. Time vs. number of vehicles with RFID 
The analysis shows that it takes 120 seconds in case of Static time intervals to pass all the 20 vehicles but in case of RFID system it takes 22 seconds to pass 20 vehicles.

\subsection{When one ambulance is detected}

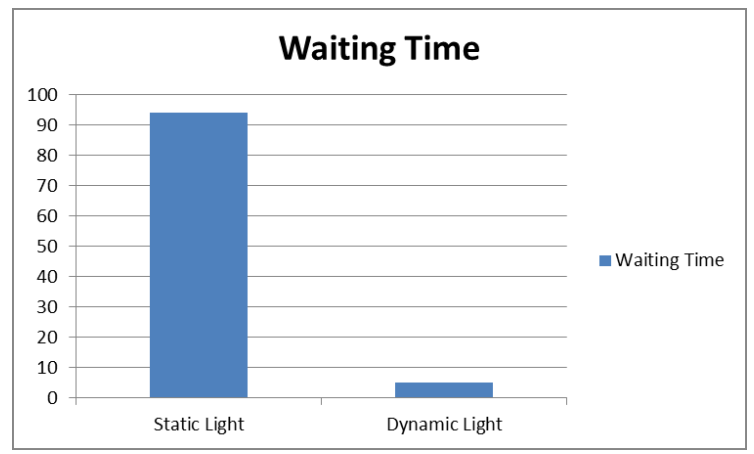

Figure 7. Waiting time for Ambulance

The time taken by the ambulance when it got stuck in the Static traffic light system was 94 seconds that is a quite large and that noticed in the Coordinated Intelligent Traffic light management system using RFID came to be 5 seconds only.

\subsection{When two ambulances are detected}

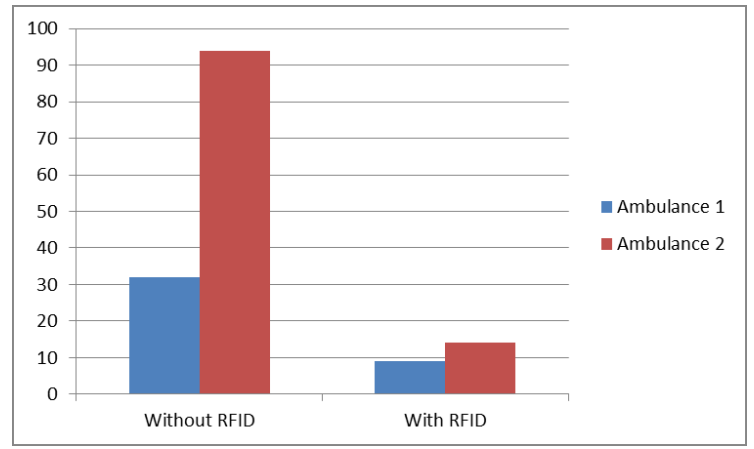

Figure 8. Waiting time for two ambulances

In the system without RFID, the first ambulance waited for 32 seconds whereas in the system with RFID, the same ambulance waited for 8 seconds. While the second ambulance without RFID system waited for 94 seconds in comparison to a waiting time of 14 seconds in case of RFID system.

\section{CONCLUSION}

The waiting time of the vehicles at the Traffic Lights has been saved by using the RFID technology. Priority has been provided to the ambulances at the Traffic Lights. The system is capable of making a decision in such a way that minimum waiting time will be required if two or more ambulances arrive at two or more different lanes at the same time. Moreover, the system can detect the congestion problems like accidents occurring at the lanes and give a warning signal to the emergency control room to take further action. The system proposed gives a highly reliable solution to the traffic problems of today and majorly serves the ones who are struggling for their lives and are in the way to hospitals but the ambulances are stuck in the traffic jams by providing them the clear pass ways.

\section{REFERENCES}

[1] Robertson, D.I. Bretherton, R.D. - Transp. \& Road Res. Lab., Crowthorne, "Optimizing networks of traffic signals in real time the SCOOTmethod", IEEE Transactions on Vehicular Technology, Vol. 40, Issue:1, Part 2, Feb 1991, pp. 11-15.

[2] Ben-Akiva, M., DePalma, A., and Kaysi, I., "Dynamic network models and driver information systems", Transportation Research Part A: General, Vol. 25, Issue 5, September 1991, pp. 251-266.

[3] Han T. and Lin C., "Design of an Intelligent Traffic Light Controller (ITLC) with VHDL", Proceeding of IEEE TENCON'02, pp. 1749-1752, 2002.

[4] Albagul A., Hrairi M., Wahyudi, Hidayathullah M.F., "Design and Development of Sensor Based Traffic Light System", American Journal of Applied Sciences 3 (3): $1745-1749,2006$

[5] Tseng S. T. and Song K. T. "Real-Time Image Tracking for Traffic Monitoring", IEEE, 5th International Conference on Intelligent Transportation Systems, Singapore, 2002.

[6] Rabie T., Shalaby A., Adbulhai B. and Rabbany A.E., "Mobile Vision-Based Vehicle Tracking and Traffic Control" IEEE, $5^{\text {th }}$ International Conference on Intelligent Transportation Systems, Singapore, 2002.

[7] Eric Ngai and Fred Riggins, "RFID: Technology, applications, and impact on business operations", International Journal of Production Economics, Vol. 112, Issue 2, April 2008- pp. 507-509.

[8] Ahmed S. Salama, Bahaa K. Saleh and Mohamad M. Eassa, "Intelligent Cross Road Traffic Management System (ICRTMS)", International Conference on Computer Technology and Development, ICCTD 2010. 